\title{
THE LOCAL RINGS OF GLOBAL DIMENSION TWO ${ }^{1}$
}

\author{
WOLMER V. VASCONCELOS
}

\begin{abstract}
A commutative local ring $A$ of global dimension two conforms to the following description: (a) If the maximal ideal $M$ is either principal or not finitely generated then $A$ is a valuation domain. (b) Otherwise $M$ is generated by a regular sequence of two elements but the ring is not necessarily noetherian. It will be noetherian if and only if it is completely integrally closed.
\end{abstract}

Introduction. This note gives a description of the commutative rings of the title. Unfortunately it is not as simple as would be desirable-that such rings be either valuation domains or noetherian rings. Nevertheless, the exceptional cases look rather as if a noetherian ring was "appended" to a valuation domain.

The problem treated here and most of the elements of the proof constitute, somehow, a delayed consequence of a lecture by Irving Kaplansky, several years ago, on rings of finite global dimension.

Preliminaries and statement of main result. Throughout $A$ will denote a commutative ring with identity. When $A$ admits a unique maximal ideal it will be called local (rather than quasi-local).

For an $A$-module $E$, the projective dimension of $E$ (proj $\operatorname{dim} E$, for short) is finite and equal to $\boldsymbol{n}$, we recall, if there is an exact sequence

$$
0 \rightarrow P_{n} \rightarrow \cdots \rightarrow P_{0} \rightarrow E \rightarrow 0
$$

with $P_{i} A$-projective and $\boldsymbol{n}$ least. Otherwise the projective dimension of $E$ is said to be infinite.

The global dimension of $A$ is then the supremum of the projective dimensions of all $A$-modules. The rings of global dimension zero are easily described-they are finite products of fields---while those of global dimension one are not so simply written-the topology of the subset of its minimal primes plays here a crucial role. However, if in addition $A$ is local, then it is simply a discrete valuation domain.

Received by the editors January 11, 1972.

AMS 1969 subject classifications. Primary 1350; Secondary 1320, 1690.

Key words and phrases. Projective dimension, global dimension, coherent ring, prime ideal, flat module, finitistic dimension.

${ }^{1}$ This research was partially supported by NSF grant GP-19995. 
THEOREM. Let $A$ be a commutative local ring of maximal ideal $M$ and global dimension two. Then:

(a) If $M$ is principal or not finitely generated, then $A$ is a valuation domain.

(b) Otherwise $M$ is generated by two elements but $A$ is not necessarily noetherian. $A$ will be noetherian if and only if it is completely integrally closed. If $A$ is not noetherian, its Krull dimension is at least three; the prime ideals which are not finitely generated are flat, countably generated, linearly ordered and contained in any finitely generated prime ideal.

Proof. It will consist of a list of more or less simple observations on $A$. First of all, note that the hypothesis is equivalent to " $A$ is not a discrete valuation domain and the ideals of $A$ have projective dimension at most one" according to [1].

(1) $A$ is a domain. In fact, if $\boldsymbol{f} \in A$ and $I=$ annihilator of $\boldsymbol{f}$, the exact sequence

$$
0 \rightarrow I \rightarrow A \rightarrow A f \rightarrow 0
$$

says that $I$ is $A$-projective and thus, by [6], free; but this is only possible if $I=(0)$.

(2) $A$ is a coherent ring. We recall that this means [4, p. 460] that finitely generated ideals are finitely presented. If $I$ is a finitely generated ideal, there exists a sequence

$$
0 \rightarrow K \rightarrow A^{n} \rightarrow I \rightarrow 0
$$

with $K$ projective and thus free of rank necessarily not greater than $\boldsymbol{n}$.

(3) $A$ is a GCD-domain, in particular $A$ is integrally closed. If $\boldsymbol{a}, \boldsymbol{b}$ are elements in $A$, there is a sequence

$$
0 \rightarrow K \rightarrow A^{2} \rightarrow(a, b) \rightarrow 0
$$

obtained by mapping $(1,0)$ onto $\boldsymbol{a}$ and $(0,1)$ onto $\boldsymbol{b} . K$ will be a free module of rank one (if $a$ or $b$ is distinct from 0 ), generated by, say, $(\alpha, \beta)$. As $(b,-a) \in K$, we can write $(b,-a)=\delta(\alpha, \beta)$. It is easily checked that $\delta$ is the greatest common divisor of $\boldsymbol{a}$ and $\boldsymbol{b}$.

We denote the GCD of $\boldsymbol{a}, \boldsymbol{b}$ by $[\boldsymbol{a}, \boldsymbol{b}]$ and use [5, p. 32] for reference. Note that if $[\boldsymbol{a}, \boldsymbol{b}]=\delta, \boldsymbol{a}=\delta \alpha, \boldsymbol{b}=\delta \beta$, then $\{\alpha, \beta\}$ form a regular sequence, i.e. $\boldsymbol{r} \alpha=\boldsymbol{s} \beta$ implies $\boldsymbol{r} \in(\beta), \boldsymbol{s} \in(\alpha)$.

(4) If $M$ is generated by one element $A$ is a valuation domain. Assume $M=(d)$; if $\boldsymbol{a}, \boldsymbol{b}$ are elements of $M,[\boldsymbol{a}, \boldsymbol{b}]=\delta$, then with $\boldsymbol{a}=\delta \alpha, \boldsymbol{b}=\delta \beta$, we must show that $\alpha$ or $\beta$ must be a unit. If it were not so $\alpha$ and $\beta$ would both be divisible by $\boldsymbol{d}$, and this is not possible by the choice of $\delta$ and (3).

Before we go on it will be necessary to recall the notion of finitistic projective dimension (FPD for brevity) of a ring $A$. It is obtained simply by 
taking, in the definition of the global dimension of $A$, those modules with finite projective dimension. We shall make use of the fact a perfect ring $A$ is one for which $\operatorname{FPD}(A)=0$ [2].

(5) If $M$ is not finitely generated $A$ is a valuation domain. We use the notation of (4). To prove the assertion it is enough to show that $I=(\alpha, \beta)$ is the unit ideal. If this is not the case $\{\alpha, \beta\}$ form a regular sequence contained in $M$. The ring $A / I$ is coherent because $A$ is coherent from (2) and $I$ is a finitely generated ideal. Also, by the change of rings theorem of [8, p. 172] applied twice, we have that the finitistic projective dimension of $A / I$ is zero. According to $[4$, p. 467] however, $A / I$ being commutative and both coherent and perfect is artinian. In particular its maximal ideal $M / I$ is finitely generated and thus $M$ will also be finitely generated.

This completes the proof of part (a) of the Theorem. Assume from now on that $M$ is finitely generated but not principal.

(6) $M$ is generated by two elements. If $M$ is minimally generated by $\boldsymbol{x}_{1}, \cdots, \boldsymbol{x}_{n}$, since proj $\operatorname{dim} M=1$, there is an exact sequence

$$
0 \longrightarrow A^{n-1} \stackrel{f}{\longrightarrow} A^{n} \longrightarrow M \longrightarrow 0 .
$$

The statement now follows from [3, p. 946] since $M$ will be generated by the $(n-1)$-minors of the matrix $f$.

(7) Example of a local ring of global dimension two which is neither a valuation domain nor a noetherian ring. For a domain $A$ of field of quotients $K$, let $A[[t)$ ) be the subring of $K[[t]]$ (=power series ring with coefficients in $K$ ) consisting of the series with constant term in $A$. If $A$ is local, $A[[t))$ is also local and in this case one checks easily that every ideal of $A[[t))$ is of the form $t^{n} J \cdot A[[t))$ where $J$ is an $A$-submodule of $K$. Now take $A$ to be a noetherian local ring of global dimension two in which $K$ has projective dimension one. (According to [7] this is equivalent to $K$ being countably generated.) A simple example is the ring $Z[x]$ of polynomials over the integers localized at the prime $(2, x)$. Then any submodule $J$ of $K$ has also projective dimension at most one. (Proof. $0 \rightarrow J \rightarrow K \rightarrow K / J \rightarrow 0$ implies the exactness of $\operatorname{Ext}_{A}^{2}(K, E) \rightarrow \operatorname{Ext}_{A}^{2}(J, E) \rightarrow 0$ for any $A$-module $E$.) Thus we get for any such $J$ a resolution

$$
0 \rightarrow F_{1} \rightarrow F_{0} \rightarrow J \rightarrow 0
$$

where $F_{0}, F_{1}$ are $A$-free. Since $A[[\boldsymbol{t}))$ is $A$-flat, one has

$$
0 \rightarrow A[[\boldsymbol{t})) \otimes_{A} F_{1} \rightarrow A[[\boldsymbol{t})) \otimes_{A} F_{0} \rightarrow J \cdot A[[\boldsymbol{t})) \rightarrow 0
$$

also exact and $A[[\boldsymbol{t})$ ) has global dimension at most two. It is obviously neither a valuation domain nor a noetherian ring.

Another way to construct the example, this time retaining the cardinality conditions, is to take the similarly defined $A[t)$ and localize it at $(M, t)$. 
This way we can apply the procedure again and obtain any number (even infinity) for the Krull dimension.

(8) Every prime ideal $P$ of $A, P \neq M$, is a directed union of principal ideals (and thus flat); $P$ is also countably generated. Indeed, if $a, b \in P$, let $\delta=$ $[a, b]$; it is enough to show that $\delta \in P$. If this were not so, with $a=\delta \alpha$, $\boldsymbol{b}=\delta \beta$ we would have $\alpha, \beta \in P$ and $P$ would contain the regular sequence $\{\alpha, \beta\}$. But for the same reasons as in $(5), A /(\alpha, \beta)$ would then be artinian and then $M=P$.

To complete the proof of the assertion we remark that proj $\operatorname{dim} P \leqq 1$ and the manner of generation of $P$ leads, as in [7], to a countable generation for $P$.

(9) Every nonfinitely generated prime ideal is contained in every finitely generated prime ideal. We have seen so far enough to conclude that the finitely generated prime ideals are either principal or $M$ itself. Let $P$ be a prime ideal not finitely generated and $(d)$ a principal prime such that $P \notin(d)$. Then we show that $P$ is finitely generated.

$A /(d)$ is a coherent domain of finitistic dimension one by (2) and $[8$, p. 172] and thus is noetherian. Consequently $(P, d)$ is a finitely generated ideal. From (8) we can assume $(P, \boldsymbol{d})=(\boldsymbol{p}, \boldsymbol{d})$ with $\boldsymbol{p} \in P$. Let $\boldsymbol{q}$ be an element of $P$ such that $p \in(q)$ but $q \notin(p)$, i.e. $p=r q, r \in M$. We can, on the other hand, write $q=\boldsymbol{t} \boldsymbol{p}+\boldsymbol{v} \boldsymbol{d}$ and hence $q=\boldsymbol{t r} q+\boldsymbol{v d}$ or $(1-\boldsymbol{t r}) q=\boldsymbol{v d}$ and $q \in(d)$. But then $P \subset(d)$.

(10) The nonfinitely generated prime ideals are linearly ordered. Let $P$, $Q$ be noncomparable, nonfinitely generated prime ideals. Let $p \in P \backslash Q$ and $\boldsymbol{q} \in Q \backslash P$. Then $\delta=[\boldsymbol{p}, \boldsymbol{q}]$ is in neither $P$ nor $Q$, and hence $P+Q$ contains $\alpha, \beta$ where $\boldsymbol{p}=\delta \alpha, \boldsymbol{q}=\delta \beta$. However, if $\boldsymbol{d} \in M \backslash M^{2},(\boldsymbol{d})$ is a prime ideal as $\boldsymbol{d}$ is indecomposable and $A$ is a GCD [5, p. 32]. Thus by (9), $P+Q \subset(d)$, which is not possible as $[\alpha, \beta]=1$.

To complete the proof of part (b) we have

(11) If $A$ is completely integrally closed it is noetherian. (The converse is trivial since, by (2), $A$ is integrally closed.) If $P$ is a prime ideal properly contained in $(d)$, with $d$ indecomposable, then clearly $P \subset \cap\left(d^{n}\right)$. Thus, if $0 \neq p \in P$ and $u=d^{-1}, p u^{n} \in A$ for all integers $n$. If $A$ is completely integrally closed then $\boldsymbol{u} \in A$, a contradiction.

REMARK. The shape of the prime spectrum of a local ring of global dimension two which is neither a valuation domain nor a noetherian ring "resembles" an umbrella. One is then tempted to call them umbrella rings.

Appendix. We shall use this section for two comments on the divisibility of flat prime ideals.

The first refers to a situation which becomes clear during the preceding proof: If $A$ is a local ring of global dimension two, then for any 
nonmaximal prime ideal $P, A_{P}$, the localization of $A$ at $P$, is a valuation domain.

Proposition A. Let $A$ be a commutative domain. Then $A$ is a valuation domain if and only if it is a GCD and the prime ideals are linearly ordered.

Proof. A valuation domain satisfies trivially the GCD condition. A ring with the above properties is local; let $\boldsymbol{a}, \boldsymbol{b}$ be elements in its maximal ideal $M$ and let $\delta=[\boldsymbol{a}, \boldsymbol{b}]$. Then with $\boldsymbol{a}=\delta \alpha, \boldsymbol{b}=\delta \beta,\{\alpha, \beta\}$ is either a regular sequence in $M$ or one of them is a unit. To show the second hypothesis to be the case, let $P$ (resp. $Q$ ) be a prime ideal minimal over $(\alpha)$ (resp. $(\beta)$ ). Say $P \subset Q$; then there is $s \notin Q$ and an integer $n$ such that $s \alpha^{n}=r \beta$. Since $\left\{\alpha^{n}, \beta\right\}$ also form a regular sequence, $s \in(\beta)$, a contradiction.

The next remark is essentially [5, p. 7, Example 5] with the finiteness hypothesis deleted.

Proposition B. (a) Let I be a flat ideal of a commutative ring $A$ and let $P$ be a prime ideal properly contained in $I$. Then $P=I \cdot P$.

(b) If $I$ is besides a prime ideal, then $J=\bigcap I^{n}$ is also a prime ideal.

Proof. (a) $I \otimes_{A} A \mid P=I / P \cdot I$ is a flat $A / P$-module and thus torsion free over $A / P$. Tensor by $A / P$ the sequence

$$
0 \rightarrow I \rightarrow A \rightarrow A / I \rightarrow 0
$$

to get

$$
0 \rightarrow \operatorname{Tor}_{1}^{A}(A|P, A| I) \rightarrow I / P \cdot I \rightarrow A / P \rightarrow A / I \rightarrow 0 .
$$

But the submodule $\operatorname{Tor}_{1}^{A}(A / P, A / I)$ of $I / P \cdot I$ is annihilated by $I / P$ and thus is trivial, i.e. $P \cap I=P \cdot I$ or $P=P \cdot I$.

(b) First note that if $\boldsymbol{x} \cdot \boldsymbol{y} \in I^{n}$ but $\boldsymbol{x} \notin I$ then $y \in I^{n}$; if $y \in I^{r} \backslash I^{r+1}, r<\boldsymbol{n}$, $I^{r}$ is a flat $A$-module and thus $I^{r} / I^{r+1}$ is $A / I$-flat and hence without torsion over $A \mid I$, a contradiction.

Assume $x \cdot y \in J=\bigcap I^{n}$ and $x \in I^{n} \backslash I^{n+1}, y \in I^{m} \backslash I^{m+1}$. By the preceding the image $\boldsymbol{x}^{\prime}$ of $\boldsymbol{x}$ in $A / I^{n+1}$ is different from 0 with annihilator contained in $I / I^{n+1}$. Now

$$
I^{m} \otimes_{A} A / I^{n+1}=I^{m} / I^{m+n+1}
$$

is a flat $A / I^{n+1}$-module, the image $y^{\prime}$ of $y$ in it is different from zero but $\boldsymbol{x}^{\prime} \cdot \boldsymbol{y}^{\prime}=0$. Thus $\boldsymbol{y}^{\prime} \in\left(\right.$ annihilator of $\left.\boldsymbol{x}^{\prime}\right) \cdot\left(I^{n} / I^{m+n+1}\right)$ by the flatness of $I^{n} / I^{m+n+1}$ and hence $y \in I^{m+1}$.

\section{REFERENCES}

1. M. Auslander, On the dimension of modules and algebras. III. Global dimension, Nagoya Math. J. 9 (1955), 67-77. MR 17, 579.

2. H. Bass, Finitistic dimension and a homological generalization of scmi-primary rings, Trans. Ames. Math. Soc. 95 (1960), 466-488. MR 28 \#12.12. 
3. L. Burch, On ideals of finite homological dimension in local rings, Proc. Cambridge Philos. Soc. 64 (1968), 941-948. MR 37 \#5208.

4. S. U. Chase, Direct product of modules, Trans. Amer. Math. Soc. 97 (1960), 457473. MR 22 \#11017.

5. I. Kaplansky, Commutative rings, Allyn and Bacon, Boston, Mass., 1970. MR 40 \#7234.

6. - Projective modules, Ann. of Math (2), 68 (1958), 372-377. MR 20 \#6453.

7. - The homological dimension of a quotient field, Nagoya Math. J. 27 (1966), 139-142. MR 33 \#2664.

8. _- Fields and rings, Univ. of Chicago Press, Chicago, Ill., 1969. MR 42 \#4345.

Department of Mathematics, Rutgers University, New Brunswick, New JERSEY 08903 